\title{
Energy characterization of Pixirad-1 photon counting detector system
}

\author{
A. Vincenzi ${ }^{b}$, P.L. de Ruvo ${ }^{a, b, d *}$, P. Delogu ${ }^{a, c}$, R. Bellazzini ${ }^{a, b}$, A. Brez ${ }^{a, b}$, M. Minuti $^{a, b}$, \\ M. Pinchera ${ }^{a, b}$, G. Spandre ${ }^{a, b}$ \\ a INFN Pisa \\ L. Bruno Pontecorvo 3, 56127 Pisa, Italy \\ ${ }^{b}$ Pixirad Imaging Counters s.r.l. \\ INFN Pisa \\ ${ }^{c}$ University of Pisa - Department of Physics "E. Fermi" \\ L. Bruno Pontecorvo 3, 56127 Pisa, Italy \\ ${ }^{d}$ University of Pisa - Department of Civil and Industrial Engineering (DICI) \\ Largo Lucio Lazzarino, 56126 Pisa, Italy \\ E-mail: luca.deruvoepi.infn.it
}

\begin{abstract}
This work is focused on the characterization of the Pixirad-1 detector system from the spectroscopic point of view. An energy calibration has been carried out using different Xray sources such as fluorescence lines, synchrotron radiation and radioactive elements. The energy resolution has been measured as function of the energy and the results have been compared with theoretical estimation. Last, the charge sharing fraction has been evaluated by exploiting the monochromatic energy of the Elettra synchrotron beam.
\end{abstract}

KEYWORDS: hybrid detectors; pixelated detectors and associated VLSI electronics; X-ray radiography and digital radiography (DR), charge transport and multiplication in solid media.

${ }^{*}$ Corresponding author. 


\section{Contents}

1. Introduction 目

2. Materials and methods 2]

2.1 X-ray sources

2.2 Acquisition of data

2.3 Raw spectra

2.4 Calibration

2.5 Energy resolution 4

2.6 Charge sharing measurements

3. Results and discussion

4. Conclusions

\section{Introduction}

Pixirad-1 (fig. (1) is the first commercial product of Pixirad Imaging Counters s.r.l., a recently constituted INFN spin-off company. The core of the X-ray imaging system is a new detector, based on chromatic photon counting, that has been realized coupling a pixelated large area ASIC, known as Pixie-II, to a matching pixelated cadmium telluride (CdTe) sensor by a flip-chip bonding technique. The ASIC is a CMOS VLSI chip with an active area of $30.7 \mathrm{~mm} \times 24.8 \mathrm{~mm}$, organized on a honeycomb matrix of $512 \times 476$ pixels. Each pixel incorporates a hexagonal electrode (top metal layer) connected to a charge amplifier, which feeds two discriminators, and two 15 bit counters. Pixels are arranged with a $60 \mu \mathrm{m}$ horizontal pitch and a vertical pitch of $51.96 \mu \mathrm{m}$. The system can operate in one color mode in which signals over a global threshold are counted. In this modality the device works as a pure photon counting detector. Moreover it is possible to select two energy bins by digitally setting up two global discriminator thresholds values, th-low and th-high, and get two images per single exposure. In this modality the device can perform the so called X-ray color imaging. To reduce unavoidable pixel to pixel DC offset variations on the discrimination level, an auto-zero or self-calibration circuit is implemented in each pixel. In this way a single global threshold per discriminator can be applied to the entire matrix [1].

In general, the main advantages of photon counting over integrating detectors are:

- the capability to select the energy threshold which determines whether a photon is counted, suppressing the noise; 


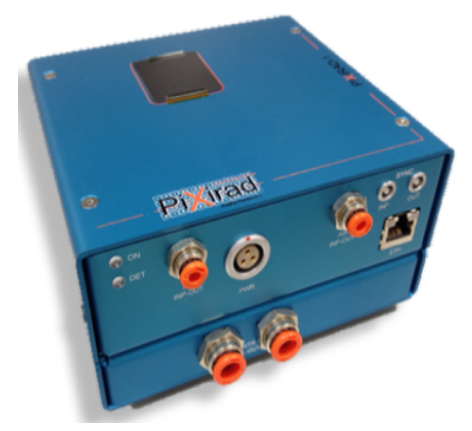

Figure 1: Pixirad-1 detector module unit

- the capability of the energy-based discrimination of photons, allowing multi-spectral imaging.

This second advantage is enhanced in the Pixirad-1 system by its dual color feature. To be spectrally useful, and to evaluate the energy resolution, a photon counting system has to be energy calibrated. The aim of the calibration is here to find the relationship between the discriminator threshold voltage $V_{t h}$ (voltage driving the threshold of the discriminator in one color mode) and the corresponding photon energy. Since the $V_{t h}$ value has the same effect on th-low and th-high in two colors mode, the calibration in one color mode has been performed.

We characterized a system with a $650 \mu$ m thick CdTe crystal, Schottky type, with electron collection on the pixels. Due to the thickness of the detector and the small size of the pixels, chargesharing effects between adjacent pixels are present. Many complex and interconnected phenomena are involved in the spatial spread of charge carriers in CdTe. Over all, the major factors influencing the charge sharing are the photo-electron range and the charge diffusion. In details, a X-ray photon absorbed in CdTe generates a photo-electron that loses energy along its track producing electronhole pairs. The cloud of charge generated around the photo-electron track represents the initial source of charge spread, which drifts under the applied electric field. This charge cloud diffuses laterally when drifting towards electrodes [2]. The range of the photo-electron and the path of the secondary charges depend on the energy of the photon absorbed, so an energy dependence of the charge sharing effects is expected. However we not have an accurate model of charge sharing in the detector and our study is based on experimental data only. Practical effect of charge sharing is that the signal due to a photon is spread over multiple pixels. Inherent energy resolution of the detector is degraded by this effect, leading to a reduced ability to discriminate between two close energies.

The calibration of thresholds represents the first part of this work. At a later stage, the energy resolution of the system has been evaluated. Moreover we studied the effect of the charge sharing from a spectroscopic point of view, quantifying it as a function of the x-ray energy of the beam.

\section{Materials and methods}

\subsection{X-ray sources}

This work has been carried out in the energy range from $3 \mathrm{keV}$ to $60 \mathrm{keV}$. Photons in the range from $3 \mathrm{keV}$ to $19 \mathrm{keV}$ have been obtained by X-ray fluorescence emissions of various materials. 


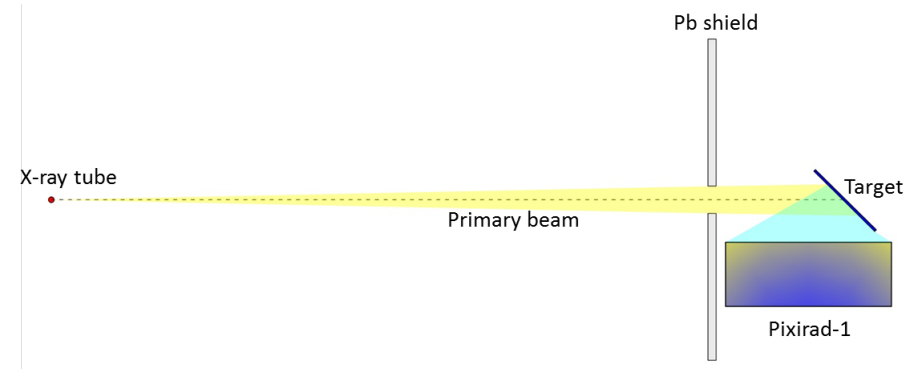

Figure 2: Set-up of fluorescence measurements

In this experimental setup a polychromatic beam produced with an X-ray tube (Hamamtsu L942102 micro focus tube) is strongly collimated on a target. This target is a thin foil of a solid element (vanadium, iron, copper, molybdenum) or a rubber balloon containing the gaseous element (argon). The target is placed close to the detector as in fig. 目.

Fluorescence is excited in the target and characteristic X-ray photons emitted by the material reach the detector. To minimize the amount of scattered photons and maximize the flux of characteristic X-ray lines, the anode voltage of the tube has been kept close to the K-edge energy of the target material. The goodness of the spectra of the radiation reaching the detector has been verified using a spectrometric device (Amptek X-123CdTe system). In the range from $8 \mathrm{keV}$ to $44 \mathrm{keV}$ synchrotron monochromatic radiation has been used. The experiment was carried on in the SYRMEP beam line at Elettra-syncrotron laboratory in Trieste (Italy). Energies in this range have been explored in steps of $2 \mathrm{keV}$. At high fluence, photon-counting detectors have nonlinear response due to dead time effect. In our experiment, the fluence has been kept as low as possible using, as attenuators, various aluminum foils of different thickness. The $59.54 \mathrm{keV}$ photons came from an Am-241 sealed source. The radioactive source was placed as close as possible to the entrance window of the detector.

\subsection{Acquisition of data}

Acquired data are arrays of counts generated in each pixel by interactions of photons with the detector during the acquisition time and can be viewed as images. The detector was operated in one color mode. For each energy, images at different $V_{t h}$ values have been taken, performing socalled thresholds scans. In a scan, the exposure time of a single image is fixed and its choice depends on the fluence of the X-ray beam reaching the detector. The result of a scan is a stack of images containing counts of signals above the corresponding values of $V_{t h}$.

\subsection{Raw spectra}

Selecting regions in the images of a stack, measuring the mean counts in those regions and plotting the obtained values as a function of $V_{t h}$ it is possible to draw integral raw spectra (see fig. 3a). Differential raw spectra are obtained from previous curves by numerical differentiation. An example of differential raw spectrum of monochromatic X-ray beams of energy lower than $26.7 \mathrm{keV}$ (K-edge of Cadmium) is shown in fig. 3 b.

In reference to figures $\mathrm{Ba}$ and $\mathrm{Bb}$, we can identify the following regions: 


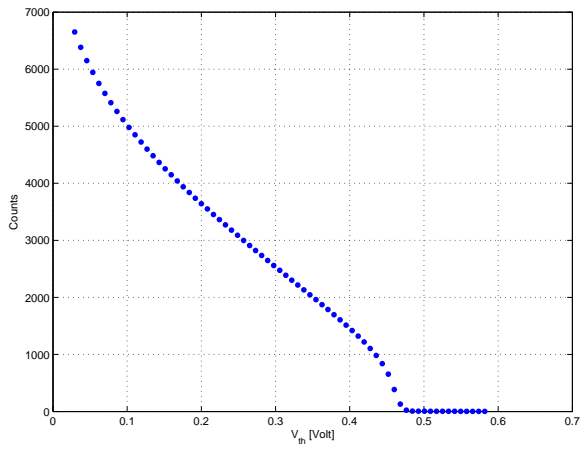

(a) Counts vs $V_{t h}$

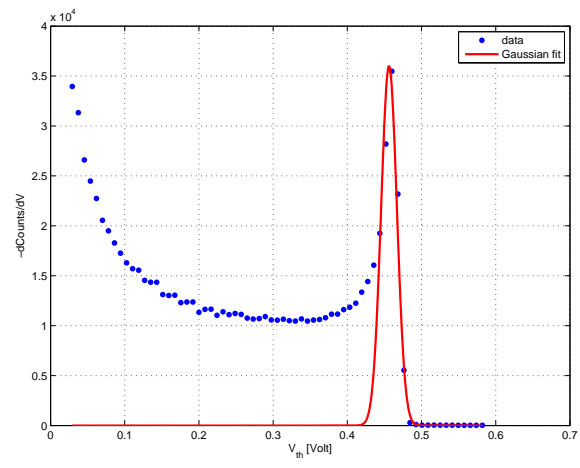

(b) Differential raw spectra

Figure 3: Data from a threshold voltage scan

1. full energy peak that appears (fig. Bb) with a distorted (on the left part) Gaussian shape: the left broadening of the peak is due to charge sharing;

2. signals corresponding to thresholds lower than the half of the photon energy: the charge shared in multiple pixels produces multiple counts;

3. signals corresponding to thresholds higher than the half of the photon energy: since charge sharing induces the spread of the signal over multiple pixels, in this region an increase in the value of $V_{t h}$ corresponds in a loss of counts.

In principle setting up a threshold value corresponding to exactly the half of photon energy one expects to avoid multiple counts and have no losses in counts.

\subsection{Calibration}

We selected type 1) regions in differential raw spectra and fitted them with Gaussian functions. The fits are done on the points belonging to the right side of the full energy peak to minimize the broadening effect due to the charge sharing. The values of $V_{t h}$ in the center of the Gaussian functions are then used as y-coordinate points in a curve where $\mathrm{x}$-coordinates are photons energies (see fig. (1). The curve has been fitted with a polynomial function and we found a relation between $V_{t h}$ and photon energy.

\subsection{Energy resolution}

After calibration, values of $V_{t h}$ in the differential raw spectra can be replaced with corresponding energy values. Linearizing the abscissas according the calibration curve, we obtained the final spectra. On these spectra, using the same fitting procedure of the section 2.4 we calculated the energy resolution. For a pixel detector an additional source of the full-peak broadening is related to slightly differences in pixel amplifier gain and the residual pixel to pixel DC offsets spread after internal compensation, we studied the energy resolution for different sizes of the region selected to extract the integral spectrum. In particular we carried on measurements on single pixels. 


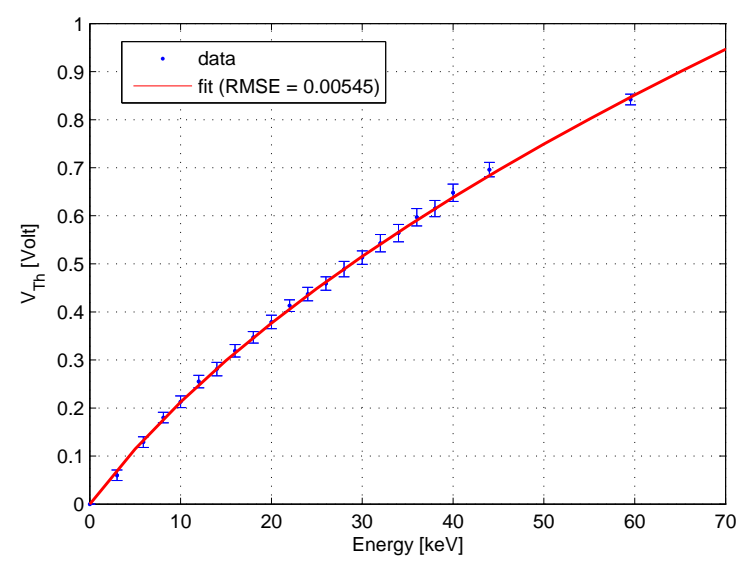

Figure 4: Energy calibration curve

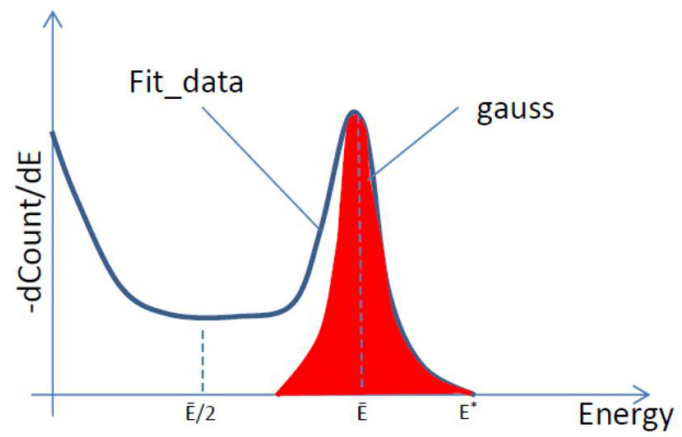

Figure 5: Sketch of a spectrum of monochromatic photon beam of energy $\bar{E}$ : the blue line represents data interpolation with cubic splines, the red area is the Gaussian integral of signal.

\subsection{Charge sharing measurements}

Starting from the shape of the spectra, it is helpful to have a method to estimate the amount of charge sharing. We did it calculating the complement to one of the ratio between the area under the Gaussian fit and the whole area under the curve starting from the half of the energy of photon. This computation has been made after interpolating the data with cubic spline, according the following relation:

$$
\text { charge sharing fraction }=1-\frac{\int \text { gauss }}{\int_{\frac{\bar{E}}{2}}^{E^{*}} \text { fitdata }}
$$

The sketch in fig. 5 shows this simple method in which $\bar{E}$ is the energy of monochromatic photon, $E^{*}$ is the end-point of spectrum. To avoid that multiple counts affect the measurements, the integral lower bound of fit-data must be equal to $\frac{\bar{E}}{2}$.

\section{Results and discussion}

The calibration curve is shown in fig. $⿴$. Referencing to the figure we highlight the absence of 


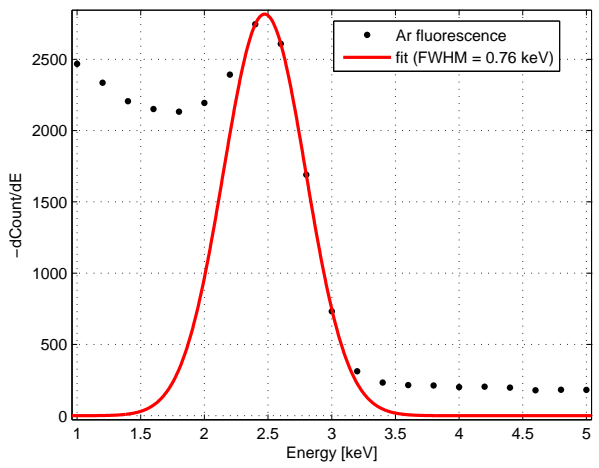

(a) Argon fluorescence line spectrum

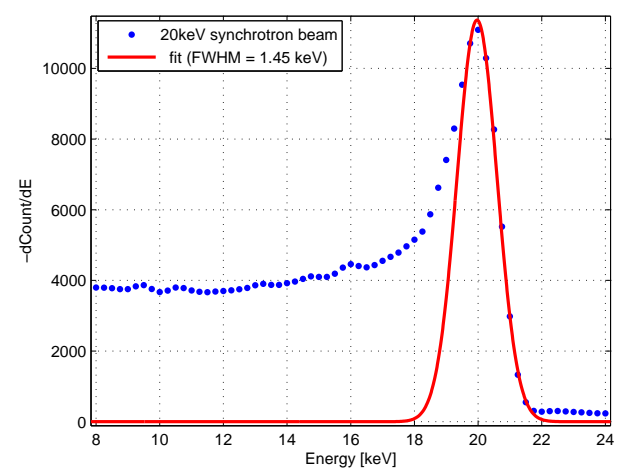

(c) $20 \mathrm{keV}$ monochromatic spectrum

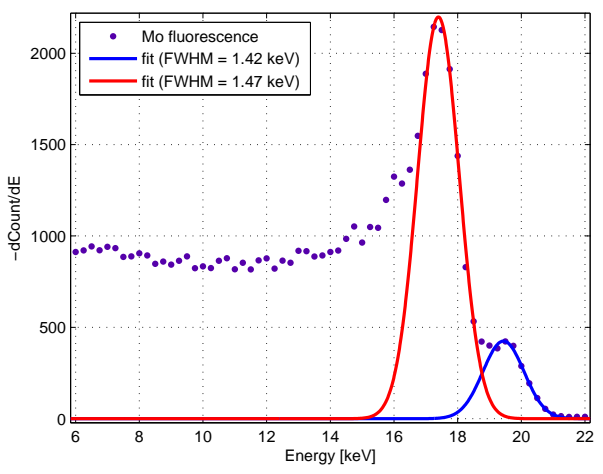

(b) Molybdenum fluorescence lines $K_{\alpha}$ and $K_{\beta}$

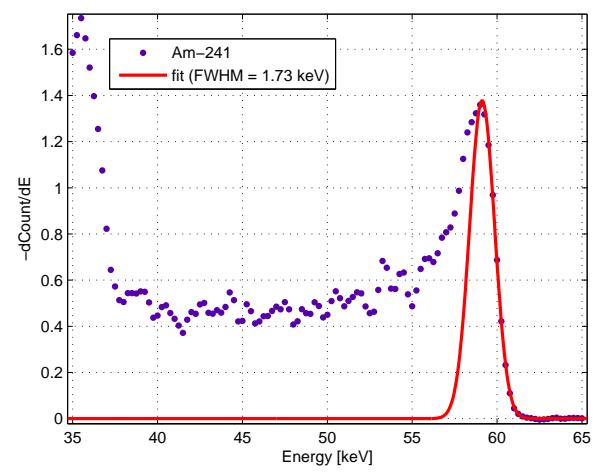

(d) Americium-241 spectrum

Figure 6: Some spectra obtained with different photon sources

saturation effects in the range up to $60 \mathrm{keV}$ making the device effective for spectroscopic studies in this range. The nonlinear behavior, occurring especially at energy greater than $15 \mathrm{keV}$, is consistent with the original design of the ASIC [四].

In fig. 6 we show some differential spectra acquired with different sources. These spectra have been obtained selecting regions composed of 1000 pixels. The good energy resolution of the system is noticeable even for very low energy photon coming from $2.96 \mathrm{keV}$ argon line fluorescence (fig. 6a), residual background of the primary beam is even present. The system is able to discriminate the $K_{\alpha}$ and $K_{\beta}$ fluorescence lines of molybdenum a $17.5 \mathrm{keV}$ and $19.6 \mathrm{keV}$ (fig. 6b). The fig. 6 plots the spectrum of $20 \mathrm{keV}$ synchrotron $\mathrm{x}$-ray beam, third-order harmonic contamination of the source and perhaps residual pileup effect cause the tail above the full energy peak. The fig. 6d shows the spectrum of americium-241 radioactive source.

The energy resolution results are summarized in tab. 1. Better spectra are expected if data come from a single pixel. The limiting factor in a single pixel analysis is the poorer statistics bringing noisier data. The differentiation of the integral spectrum propagates that noise.

However some single pixel spectra have been obtained (see fig. (7) proving the expected improvement of the energy resolution. In the simplest model, the energy resolution of the detector is influenced only by electronic and statistical noise [3]: 
Table 1: Energy resolution on a cluster of 1000 pixels

\begin{tabular}{ccc}
\hline Energy [keV] & FWHM [keV] & Resolution \% \\
\hline 2.96 & 0.76 & 25.8 \\
4.95 & 0.85 & 17.1 \\
8.1 & 0.88 & 10.8 \\
10 & 0.92 & 9.2 \\
12 & 1.06 & 8.8 \\
14 & 1.20 & 8.6 \\
16 & 1.31 & 8.2 \\
18 & 1.48 & 8.2 \\
22 & 1.38 & 6.3 \\
38 & 1.60 & 4.2 \\
59.54 & 1.73 & 2.9 \\
\hline
\end{tabular}

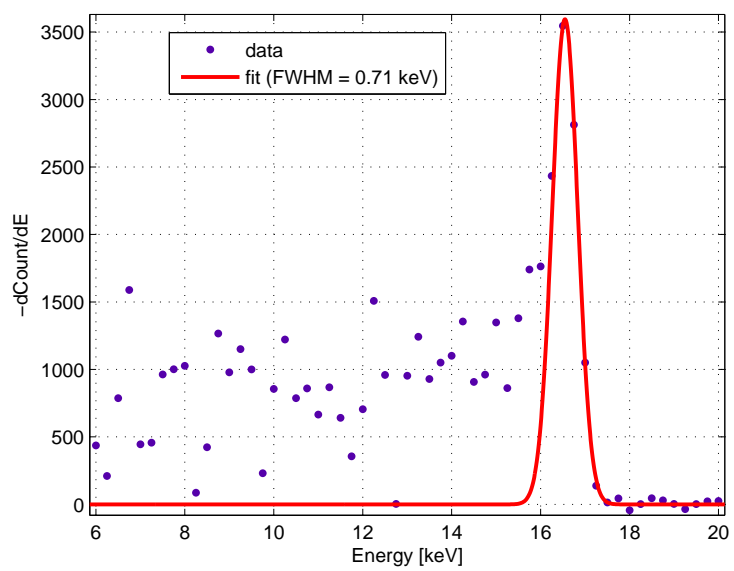

Figure 7: Single pixel energy resolution @ $16 \mathrm{keV}$ (monochromatic energy)

$$
F W H M_{\text {overall }}^{2}=F W H M_{\text {el }}^{2}+F W H M_{\text {stat }}^{2}
$$

The theoretical energy resolution based on eq. 3.1 for a photon of $16 \mathrm{keV}$, considering that the Equivalent Noise Charge of ASIC is $50 e^{-}$RMS and assuming a Fano factor for CdTe of 0.15 [凹], is $574 \mathrm{eV}$. The spectrum in fig. $\square$ shows the measured FWHM $(710 \mathrm{eV})$ at the same energy. This value can be considered close to the theoretical one keeping in mind that eq. 3.1 does not take into account the worsening influence of coupling capacitance between the CdTe sensor and the ASIC. The broadening effect on energy resolution of cluster of pixels (1310 eV @ $16 \mathrm{keV})$ compared to the single pixel (710 eV @ $16 \mathrm{keV}$ ) one is due to small differences of amplifier gain (of the order of $2 \%$ rms) and residual offset variation from pixel to pixel which are not completly corrected by the self-calibration. In fig. 8 we show the fraction of charge shared events, calculated according the eq. 2.1, for monochromatic photon beams in the energy range from $3 \mathrm{keV}$ to $26 \mathrm{keV}$, below the 


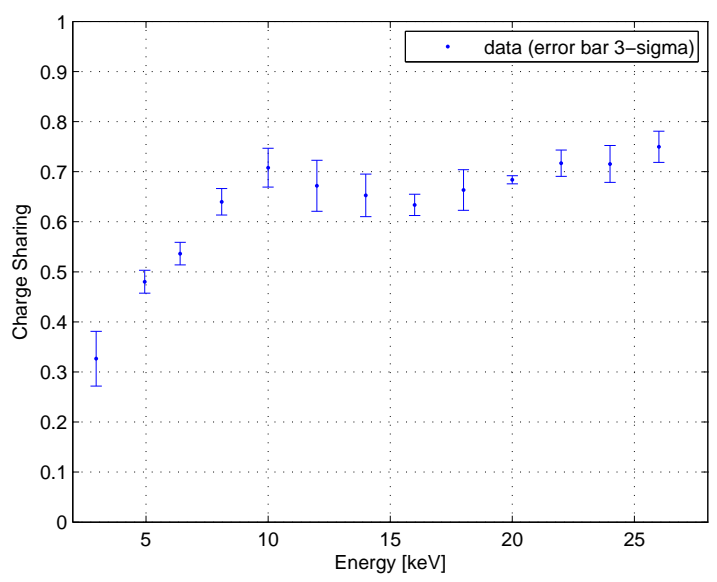

Figure 8: Fraction of charge shared events measured at different energy

energy of the K-edge of the Cadmium. The plot suggests that up to $10 \mathrm{keV}$ the fraction of charge sharing events increase almost linearly and after a knee it reaches a value of about $70 \%$, further studies are ongoing to better understand this behavior.

\section{Conclusions}

A Pixirad-1 calibration curve has been obtained in a wide energy range (from 3 to $60 \mathrm{keV}$ ). The detector has shown good energy resolution (from $25,8 \%$ to $2,9 \%$ in the same energy range). The statistical fluctuations affect the energy resolution according to the theoretical estimation. The agreement is better in case of single pixel than in case of cluster of pixels. The source of the FWHM broadening effect for cluster of pixels is related to slightly differences in pixel amplifier gain and the residual DC offsets after compensation. As expected the sharpness of color separation is limited by charge sharing effects, when working with polychromatic beams, and using the simple method described this contribution proves to be non-negligible.

\section{Acknowledgments}

We desire to thank all the staff of Syrmep beam line at Elettra synchrotron in Trieste.

\section{References}

[1] R. Bellazzini et al., Chromatic X-Ray imaging with a fine pitch CdTe sensor coupled to a large area photon counting pixel ASIC, 2013 JINST 8 C02028

[2] X Wang et al., MicroCT with energy-resolved photon-counting detectors, Phys. Med. Biol. 56(2011) page 2791

[3] G.F. Knoll, Radiation detection and measurements, John Wiley and Sons, Inc., New York 2000

[4] Rossi, Fischer, Rohe, Wermes, Pixel Detectors: From Fundamentals to Applications, Springer 2006 